\title{
COMPUTATION OF REALIZATIONS COMPOSED OF DYNAMIC AND STATIC PARTS OF IMPROPER TRANSFER MATRICES
}

\author{
TADEUSZ KACZOREK \\ Faculty of Electrical Engineering, Białystok Technical University \\ ul. Wiejska 45D, 15-351 Białystok \\ e-mail:kaczorek@isep.pw.edu.pl
}

\begin{abstract}
The problem of computing minimal realizations of a singular system decomposed into a standard dynamical system and a static system of a given improper transfer matrix is formulated and solved. A new notion of the minimal dynamical-static realization is introduced. It is shown that there always exists a minimal dynamical-static realization of a given improper transfer matrix. A procedure for the computation of a minimal dynamical-static realization for a given improper transfer matrix is proposed and illustrated by a numerical example.
\end{abstract}

Keywords: minimal realization, decomposition, improper transfer matrix, singular linear system

\section{Introduction}

The computation of a minimal realization for a given transfer matrix is one of the classical problems in control theory. There exist many well-known methods for the computation of minimal realizations for given proper and improper transfer matrices (Christodoulou and Mertzios, 1985; Kaczorek, 1992; Kailath, 1980; Roman and Bullock, 1975; Sinha Naresk, 1975; Wolovich and Guidorsi, 1977). It is also well known that a singular linear system described by static equations can be decomposed into two subsystems, a standard dynamical subsystem and a static subsystem (Kaczorek, 1992). The main purpose of this paper is to propose a method for the computation of minimal realizations of a singular system decomposed into a standard dynamical system and a static system of a given improper transfer matrix. A new notion of the minimal dynamical-static realization will be introduced. It will be shown that there always exists a minimal dynamical-static realization of a given improper transfer matrix. A procedure for the computation of a minimal dynamical-static realization of a given improper transfer matrix will be proposed.

To the best of the author's knowledge, the problem of computing a minimal dynamical-static realization for a given improper transfer matrix has not been considered yet.

\section{Preliminaries and problem formulation}

Let $\mathbb{R}^{n \times m}$ be the set of $n \times m$ real matrices and $\mathbb{R}^{n}:=$ $\mathbb{R}^{n \times 1}$. Consider the singular continuous-time linear system

$$
\begin{aligned}
E \dot{x} & =A x+B u, \\
y & =C x,
\end{aligned}
$$

where $x \in \mathbb{R}^{n}, u \in \mathbb{R}^{m}, y \in \mathbb{R}^{p}$ are respectively the state vector, the input vector and the output vector, and $E, A \in \mathbb{R}^{n \times n}, B \in \mathbb{R}^{n \times m}, C \in \mathbb{R}^{p \times n}$. It is assumed that $\operatorname{det} E=0$ and

$$
\operatorname{det}[E s-A] \neq 0
$$

for some $s \in \mathbb{C}$ (the field of complex numbers).

It is well known (Kaczorek, 1992) that the singular system (1) can be decomposed into the standard dynamical system

$$
\begin{aligned}
& \dot{x}_{1}=A_{1} x_{1}+B_{1} u, \\
& y_{1}=C_{1} x_{1},
\end{aligned}
$$

and the static system

$$
\begin{aligned}
& x_{2}=A_{21} x_{1}+B_{20} u+B_{21} \dot{u}++B_{2 r} u^{(r)}, \\
& y_{2}=C_{2} x_{2},
\end{aligned}
$$

such that

$$
y=y_{1}+y_{2}, \quad\left[\begin{array}{l}
x_{1} \\
x_{2}
\end{array}\right]=Q x, \quad \operatorname{det} Q \neq 0
$$


(often $Q=I$ ), where $x_{1} \in \mathbb{R}^{n_{1}}, x_{2} \in \mathbb{R}^{n_{2}}, n_{1}+n_{2}=$ $n, A_{1} \in \mathbb{R}^{n_{1} \times n_{1}}, B_{1} \in \mathbb{R}^{n_{1} \times m}, C_{1} \in \mathbb{R}^{p \times n_{1}}, A_{21} \in$ $\mathbb{R}^{n_{2} \times n_{1}}, B_{2 k} \in \mathbb{R}^{n_{2} \times m}$ for $k=0,1, \ldots, r$ and $u^{(r)}=$ $\mathrm{d}^{r} u / \mathrm{d} t^{r}$.

The decomposition can be obtained using the modified shuffle algorithm (Kaczorek, 1992).

Lemma 1. The transfer matrix of the singular system decomposed into the standard dynamical system (3) and the static system (4) is given by

$$
\begin{aligned}
T(s)= & \left(C_{1}+C_{2} A_{21}\right)\left[I_{n_{1}} s-A_{1}\right]^{-1} B_{1} \\
& +C_{2}\left(B_{20}+B_{21} s+\cdots+B_{2 r} s^{r}\right) .
\end{aligned}
$$

Proof. From (3a) and (4a) we have

$$
\begin{aligned}
{\left[\begin{array}{l}
X_{1} \\
X_{2}
\end{array}\right]=} & {\left[\begin{array}{cc}
{\left[I_{n_{1}} s-A_{1}\right]} & 0 \\
-A_{21} & I_{n_{2}}
\end{array}\right]^{-1} } \\
& \times\left[\begin{array}{c}
B_{1} \\
B_{20}+B_{21} s+\cdots+B_{2 r} s^{r}
\end{array}\right] U,(7)
\end{aligned}
$$

where $X_{k}=X_{k}(s)=L\left[x_{k}(t)\right], U=U(s)=L[u(t)]$ are the Laplace transforms of $x_{k}$ and $u$, respectively.

Taking into account that

$$
\left[\begin{array}{cc}
{\left[I_{n_{1}} s-A_{1}\right]} & 0 \\
-A_{21} & I_{n_{2}}
\end{array}\right]^{-1}=\left[\begin{array}{cc}
{\left[I_{n_{1}} s-A_{1}\right]^{-1}} & 0 \\
A_{21}\left[I_{n_{1}} s-A_{1}\right]^{-1} & I_{n_{2}}
\end{array}\right],
$$

from (3b), (4b) and (5) we obtain for the Laplace transform of $y$,

$$
\begin{aligned}
Y= & {\left[\begin{array}{ll}
C_{1} & C_{2}
\end{array}\right]\left[\begin{array}{c}
X_{1} \\
X_{2}
\end{array}\right] } \\
= & {\left[\begin{array}{ll}
C_{1} & C_{2}
\end{array}\right]\left[\begin{array}{cc}
{\left[I_{n_{1}} s-A_{1}\right]^{-1}} & 0 \\
A_{21}\left[I_{n_{1}} s-A_{1}\right]^{-1} & I_{n_{2}}
\end{array}\right] } \\
& \times\left[\begin{array}{c}
B_{1} \\
B_{20}+B_{21} s+\cdots+B_{2 r} s^{r}
\end{array}\right] U \\
= & {\left[\left(C_{1}+C_{2} A_{21}\right)\left[I_{n_{1}} s-A_{1}\right]^{-1} B_{1}\right.} \\
& \left.+C_{2}\left(B_{20}+B_{21} s+\cdots+B_{2 r} s^{r}\right)\right] U .
\end{aligned}
$$

Formula (6) follows from (8).

Definition 1. The matrices $A_{1}, A_{21}, B_{1}, B_{20}$, $B_{21}, \ldots, B_{2 r}, C_{1}, C_{2}$ constitute a dynamical-static realization of an improper transfer matrix $T(s)$ if they satisfy (6). A realization is called minimal if the matrices $A_{1}$ and $A_{21}$ have minimal dimensions among all realizations of $T(s)$.

The realization problem can be stated as follows: Given an improper transfer matrix $T(s) \in \mathbb{R}^{p \times m}(s)$ (the set of $p \times m$ rational matrices in $s$ ), find a dynamical-static realization of a given improper transfer matrix $T(s)$.
In what follows, a procedure for the computation of a minimal dynamical-static realization of a given improper transfer matrix will be proposed.

\section{Problem Solution}

Any given improper transfer matrix $T(s) \in \mathbb{R}^{p \times m}(s)$ can be decomposed into the polynomial part

$$
P(s)=P_{0}+P_{1} s+\cdots+P_{r} s^{r}
$$

and the strictly proper part $T_{s p}(s)$, i.e.,

$$
T(s)=P(s)+T_{s p}(s) .
$$

From the comparison of (6) and (10), we have

$$
\begin{aligned}
P(s) & =P_{0}+P_{1} s+\cdots+P_{r} s^{r} \\
& =C_{2}\left(B_{20}+B_{21} s+\cdots+B_{2 r} s^{r}\right)
\end{aligned}
$$

and

$$
T_{s p}(s)=\left(C_{1}+C_{2} A_{21}\right)\left[I_{n_{1}} s-A_{1}\right]^{-1} B_{1} .
$$

Using one of the well-known methods (Christodoulou and Mertzios, 1985; Kaczorek, 1992; Kailath, 1980; Roman and Bullock, 1975; Sinha Naresk, 1975; Wolovich and Guidorsi, 1977), we can determine a minimal realization $A_{1}, B_{1}, \bar{C}_{1}$ of $T_{s p}(s)$ satisfying

$$
\bar{C}_{1}\left[I_{n_{1}} s-A_{1}\right]^{-1} B_{1}=T_{s p}(s) .
$$

Given the matrices $P_{k}, \quad k=0,1, \ldots, r$ and $A_{1}, B_{1}, \bar{C}_{1}$, in order to solve the realization problem, we have to find the matrices $A_{1}, A_{21}, B_{1}, B_{2 k}, k=0,1, \ldots, r$ and $C_{1}$ and $C_{2}$ satisfying

$$
C_{1}+C_{2} A_{21}=\bar{C}_{1}, \quad C_{2} B_{2 k}=P_{k}
$$

for $k=0,1, \ldots, r$.

Note that there exist many matrices $A_{21}, C_{1}, C_{2}$ and $B_{2 k}, k=0,1, \ldots, r$ satisfying (14) for given $\bar{C}_{1}$ and $P_{k}, k=0,1, \ldots, r$. One way to find the desired matrices is to choose first $C_{2}$ and $A_{21}$ (or $C_{1}$ and $C_{2}$ ) and compute $C_{1}$ (or $A_{21}$ ) and $B_{2 k}, k=0,1, \ldots, r$ from (14). Therefore, we can compute a minimal dynamical-static realization of a given improper transfer matrix $T(s) \in \mathbb{R}^{p \times m}(s)$ using the following procedure:

\section{Procedure 1.}

Step 1. Decompose a given transfer matrix $T(s)$ into the polynomial part (9) and the strictly proper part $T_{s p}(s)$.

Step 2. Using one of the well-known methods compute a minimal realization $A_{1}, B_{1}, \bar{C}_{1}$ of $T_{s p}(s)$. 
Step 3. Choose the matrices $C_{2}, A_{21}$ (or $C_{1}$ and $C_{2}$ ) and, using (14), compute the matrices $B_{2 k}, k=$ $0,1, \ldots, r$ and $C_{1}$ (or $A_{21}$ ).

Remark 1. The dimensions of the matrices $B_{2 k}, k=$ $0,1, \ldots, r$ and $C_{2}$ are determined by the dimension $m \times p$ of the transfer matrix $T(s)$. A dynamical-static realization of $T(s)$ is minimal if and only if the realization $A_{1}, B_{1}, \bar{C}_{1}$ of $T_{s p}(s)$ is minimal.

From the above discussion we have the following result:

Theorem 1. For a given improper transfer matrix $T(s) \in$ $\mathbb{R}^{p \times m}(s)$ there always exists a minimal dynamical-static realization $A_{1}, A_{21}, B_{1}, B_{2 k}, k=0,1, \ldots, r, C_{1}$ and $C_{2}$. This realization can be computed using Procedure 1 .

Example 1. Find a minimal dynamical-static realization of the transfer matrix

$$
T(s)=\left[\begin{array}{cc}
\frac{s^{3}+s^{2}+1}{s} & \frac{s^{2}+2 s+3}{s+1} \\
\frac{2 s^{2}+4 s+2}{s+2} & \frac{s^{3}+2 s^{2}+s+3}{s+2}
\end{array}\right] .
$$

Using Procedure 1, we obtain the following: Step 1. The transfer matrix (15) can be decomposed into the polynomial part

$$
\begin{aligned}
P(s) & =\left[\begin{array}{cc}
s^{2}+s & s+1 \\
2 s & s^{2}+1
\end{array}\right] \\
& =\left[\begin{array}{ll}
0 & 1 \\
0 & 1
\end{array}\right]+\left[\begin{array}{ll}
1 & 1 \\
2 & 0
\end{array}\right] s+\left[\begin{array}{ll}
1 & 0 \\
0 & 1
\end{array}\right] s^{2} \\
& =P_{0}+P_{1} s+P_{2} s^{2}
\end{aligned}
$$

and the strictly proper part

$$
T_{s p}(s)=\left[\begin{array}{cc}
\frac{1}{s} & \frac{2}{s+1} \\
\frac{2}{s+2} & \frac{1}{s+2}
\end{array}\right] .
$$

Step 2. A minimal realization of (17) has the form

$$
\begin{gathered}
A_{1}=\left[\begin{array}{ccc}
0 & 0 & 1 \\
0 & -2 & 0 \\
0 & 0 & -1
\end{array}\right], \\
B_{1}=\left[\begin{array}{cc}
1 & 2 \\
2 & 1 \\
0 & -2
\end{array}\right], \quad \bar{C}_{1}=\left[\begin{array}{lll}
1 & 0 & 0 \\
0 & 1 & 0
\end{array}\right],
\end{gathered}
$$

Step 3. In this case we choose, e.g.,

$$
C_{2}=\left[\begin{array}{ll}
1 & 0 \\
0 & 1
\end{array}\right], \quad A_{21}=\left[\begin{array}{ccc}
0 & -1 & 0 \\
-1 & 0 & -1
\end{array}\right] .
$$

Then from (14) we obtain

$$
\begin{gathered}
C_{1}=\bar{C}_{1}-C_{2} A_{21}=\left[\begin{array}{lll}
1 & 1 & 0 \\
1 & 1 & 1
\end{array}\right], \\
B_{20}=P_{0}=\left[\begin{array}{ll}
0 & 1 \\
0 & 1
\end{array}\right], \quad B_{21}=P_{1}=\left[\begin{array}{ll}
1 & 1 \\
2 & 0
\end{array}\right], \\
B_{22}=P_{2}=\left[\begin{array}{ll}
1 & 0 \\
0 & 1
\end{array}\right] .
\end{gathered}
$$

The desired minimal dynamical-static realization of the transfer matrix (15) is given by (18)-(20).

\section{Concluding Remarks}

The problem of computing a minimal realization of a singular system decomposed into the standard dynamical system (3) and the static system (4) of a given improper transfer matrix was formulated and solved. A new notion of the minimal dynamical-static realization of a given transfer matrix was introduced. It was shown that there always exist a minimal dynamical-static realization of a given improper transfer matrix. A procedure for computing a minimal dynamical-static realization of a given improper transfer matrix was proposed and illustrated by a numerical example. With slight modifications (by substitution of $s$ by $z$ and of the derivative by the shifting operator) the proposed method can be extended to discrete-time linear systems.

\section{Acknowledgment}

The work was supported by the Ministry of Science and Higher Education under grant no. 3T11A00627.

\section{References}

Christodoulou M.A. and Mertzios B.G. (1985): Realization of singular systems via Markov parameters. - Int. J. Contr., Vol. 42, No. 6, pp. 1433-1441.

Kaczorek T. (1992): Linear Control Systems, Vol. 1. - New York: Wiley.

Kailath T. (1980): Linear Systems. — Englewood Cliffs: Prentice-Hall

Roman J.R. and Bullock T.E. (1975): Minimal partial realization in canonical form. - IEEE Trans. Automat. Contr., Vol. AC-20, No. 4, pp. 529-533.

Sinha Naresk K. (1975): Minimal realization of transfer function matrices: A comparative study of different methods. - Int. J. Contr., Vol. 22, No. 5, pp. 627-639.

Wolovich W.A. and Guidorsi R. (1977): A general algorithm for determining state-space representations. - Automatica, Vol. 13, pp. 295-199.

Received: 20 December 2006 